\title{
Germanica
}

GERMANICA

$53 \mid 2013$

Littérature et cinéma dans l'espace germanophone contemporain : jeux intermédiaux, modes de transfert, adaptations

\section{Ironie im Medienwechsel}

Irony in media transposition

L'ironie dans la transposition médiatique

Jens Liebich

\section{(2) OpenEdition}

Journals

Édition électronique

URL : http://journals.openedition.org/germanica/2271

DOI : 10.4000/germanica.2271

ISSN : 2107-0784

Éditeur

Université de Lille

Édition imprimée

Date de publication : 30 décembre 2013

Pagination : 13-27

ISBN : 9782913857322

ISSN : 0984-2632

Référence électronique

Jens Liebich, «Ironie im Medienwechsel », Germanica [Online], 53 | 2013, Online erschienen am: 04 Februar 2014, abgerufen am 06 Oktober 2020. URL : http://journals.openedition.org/germanica/2271 ; DOI : https://doi.org/10.4000/germanica.2271

(c) Tous droits réservés 
D'un médium à l'autre : jeux intermédiaux et modes de transfert 



\title{
Ironie im Medienwechsel
}

\author{
Jens LIEBICH \\ Université de Nantes
}

Der engste Verwandte des Films ist laut James Monaco der Roman, denn beide ,erzählen [...] lange Geschichten mit einer Fülle von Details, und sie tun dies aus der Perspektive des Erzählers, der oft eine gewisse Ironie zwischen Geschichte und Betrachter gibt" 1 . Diese Gemeinsamkeiten sind jedoch im Roman bzw. im Film unterschiedlich verteilt. Viele Handlungsdetails gehen bei Verfilmungen in der Regel verloren, jedoch kann der Film versuchen, sprachlich vermittelte Beschreibungen ins Visuelle zu übersetzen. Eben darin sieht Monaco den wesentlichen Unterschied: „Romane werden vom Autor erzählt. Wir sehen und hören nur, was er uns sehen und hören lassen möchte. Filme werden auch von ihren Autoren erzählt, aber wir sehen und hören sehr viel mehr als das, was ein Regisseur notwendigerweise möchte" 2 . Damit soll auf eine gewisse Freiheit hingewiesen werden, die der Zuschauer dem Leser gegenüber besäße. Monaco versteht alle Beschreibungen des Romanciers als durch Blickwinkel, Vorurteile und Sprache des Erzählers gefilterte, wohingegen der Film ein gewisses $\mathrm{Ma}$ an Freiheit lasse, auszuwählen und die Aufmerksamkeit auf dieses oder jenes Detail zu lenken ${ }^{3}$.

Diese Unterscheidung erscheint nachvollziehbar, doch ist sie ungenügend. Gefiltert ist die Beschreibung des Romans, wie es die

1. - James Monaco, Film verstehen. Kunst, Technik, Sprache, Geschichte und Theorie des Films und der neuen Medien, Hamburg, Rowohlt, 5. Auflage, 2004, S. 45.

2. - Ebd., S. 46.

3. - Vgl. ebd., S. 45. 
Darstellung des Films ist: Kameraperspektive, Schnitt, Licht, Dekor, Kostüme, Ton und Musik bestimmen das Bild. Dies kann man nun als Filterung oder als künstlerische und dem Medium entsprechende Gestaltung verstehen. Nicht nur ergänzen, sondern widersprechen muss man Monacos Behauptung: „Die Worte auf der Buchseite sind immer dieselben, aber das Bild auf der Leinwand ändert sich ständig, je nachdem, wohin wir unsere Aufmerksamkeit lenken. Der Film ist, so gesehen, eine sehr viel reichere Erfahrung"4. Hier spricht der Filmwissenschaftler. Die Worte der Buchseite sind im materiellen Sinn immer dieselben, so wie es jedes einzelne Bild aber auch ist, doch wie die Worte verstanden werden, wie sie wirken, ist abhängig von vielen Faktoren. Alter, Geschlecht, Erziehung, Bildung, Interessen, Kulturkreis etc. bestimmen unser Verständnis, so dass uns gute Bücher bei jeder Lektüre etwas Neues erkennen lassen. Während des Lesens ist zudem jeder Leser Regisseur des eigenen Kopfkinos, womit ein Buch eben in der Vorstellung Bilder produziert, was ein Film eher selten vermag 5 . Die beim Zuschauen aufgenommenen Bilder eines Films werden nicht in dem Maß produktiv weiterverarbeitet, wie es bei den Worten eines Textes geschieht. Die bereits vorhandenen Filmbilder werden im Kopf des Zuschauers nicht durch eigene ergänzt, somit könnte eher das Buch die reichere Erfahrung sein.

Die besondere und auch von Monaco anerkannte Stärke des Romans scheint das Spiel mit Worten zu sein, die ,subtilen und komplexen Ironien der Sprache"6. Zugleich sieht er die Ironie zwischen Geschichte und Betrachter als Gemeinsamkeit beider Medien. An einer Literaturverfilmung soll exemplarisch untersucht werden, wie sich der Humor, speziell die Ironie, durch den Wechsel vom Buch zum Film verändert und wie diese Veränderungen Auswirkungen auf die gesamte Erzählung haben.

Um die Veränderungen erkennen und beschreiben zu können, soll vor allem das „Wie“ - also die Darstellung - und nur beiläufig das „Was“ - also die Handlung und die erzählte Welt - in Buch und Film untersucht und verglichen werden. Wenngleich im Film kein Sprachsystem vorhanden ist, da er keine kodifizierte Grammatik, kein gelistetes Vokabular und auch keine Gebrauchsregeln hat, übt er „dennoch viele der Kommunikations-Funktionen der Sprache aus"7 und kann anhand dieser mit einem Text verglichen werden. Monaco

4. - Ebd., S. 47.

5. - Dass es zumindest punktuell möglich ist, zeigt die bekannte Duschszene in Alfred Hitchcocks Film Psycho (1960), die das Grauen vor allem dadurch erschafft, dass sie es im Kopf der Zuschauer entstehen lässt, es aber nicht zeigt.

6. - Ebd.

7. - Ebd., S. 61. 
verweist zur Filmanalyse auf das Zeichensystem und die Codes eines Films, wobei letztere auch anderen Kommunikationssystemen entlehnt sein können. So ließe sich jeder musikalische Code zum Beispiel in der Filmmusik benutzen ${ }^{8}$. Aber können Wortspielereien, Humor eines Textes im Allgemeinen und die Ironie im Besonderen verfilmt werden? Auf welche Weise wird etwas ausgedrückt und wie verändert sich das Ausgedrückte durch den Medienwechsel? Jens Sparschuhs Der Zimmerspringbrunnen soll exemplarisch daraufhin untersucht werden.

Die Handlung des bekanntesten Romans von Jens Sparschuh, der 1995 erschienen ist und sogar als einer der erfolgreichsten Kandidaten für den vielfach eingeforderten Wenderoman gesehen wird ${ }^{9}$, ist schnell erzählt. Hinrich Lobek, der Protagonist und Ich-Erzähler der Geschichte, arbeitete in der ,Kommunalen Wohnungsverwaltung“, wurde aber nach der Wende ,abgewickelt“ (S. 10) ${ }^{10}$ und ist nun seit über drei Jahren arbeitslos. Er ist zu jung, um in Rente zu gehen, aber zu alt, um unter Millionen Arbeitslosen als geeigneter Bewerber für einen beruflichen Neuanfang zu gelten. Die Zeit der Arbeitslosigkeit hinterlässt deutliche Spuren: Isoliert von der Gesellschaft lassen seine kommunikativen Fähigkeiten nach, gefangen in seinen Desillusionertheit gelingt es ihm nicht, Stabilität und Ordnung in der sich wandelnden Welt zu finden, und durch sein egozentrisches Weltbild entfernt er sich zunehmend von seiner beruflich erfolgreichen Frau ${ }^{11}$. Das „HALLO-BERLINWochenhoroskop" (S. 7) animiert ihn zur Eigeninitiative und er bewirbt sich auf die Vertreterstelle der oberrheinischen Firma „PANTA RHEIn“ (Vgl. S. 10f.). Nach unerwartet erfolgreicher Bewerbung versucht Lobek mit dem Verkauf von Zimmerspringbrunnen zurück ins Leben $\mathrm{zu}$ finden. Als sein vernachlässigter Hund durstend das Wasser eines Modells aussäuft, brennt dieses durch und muss von Lobek repariert werden. Die notgedrungenen Umarbeitungen machen ihn zum Erfinder von „Atlantis“ - einem Zimmerspringbrunnen mit dem Grundriss der DDR, in dessen Mitte ein Kugelschreiber als Fernsehturm wasserspritzend emporsteigt. Der berufliche Erfolg gibt ihm den lang ersehnten Halt in einer sich rasch verändernden Gesellschaft, doch seine Ehe mit Julia scheitert - vermutlich endgültig, das lässt der Roman offen.

Beruflicher Erfolg und privates Scheitern sind im Zimmerspringbrunnen treibende Kräfte der Erzählung. Ursächlich

8. - Vgl. ebd., S. 61f.

9. - Vgl. Frank Thomas Grub, ,Wende“ und „Einheit“ im Spiegel der deutschsprachigen Literatur, Bd. 1 Untersuchungen, Berlin, de Gruyter, 2003, S. 384.

10. - Seitenangaben erfolgen hier und im Folgenden direkt im Text mit Angabe der Seitenzahl in Klammern nach dieser Ausgabe: Jens Sparschuh, Der Zimmerspringbrunnen, Berlin, Goldmann Verlag, 1997.

11. - Vgl. Jill E. Twark, Humor, Satire and Identity: Eastern German Literature in the 1990s, Berlin, de Gruyter, 2007, S. 59. 
für beide ist der Gebrauch bzw. Nichtgebrauch der Sprache, was zu Missverständnissen führt, durch die die Handlung vorangetrieben wird. Die Krise Hinrich Lobeks ist eine Identitätskrise, die sich auch als Sprachkrise äußert:

Sogar Julia fiel das auf: Mit mir könne man nicht mehr reden, ihr fehle der Austausch mit mir. Da könnte man sich gleich vor ein Aquarium setzen. - Soweit ihre Darstellung.

Richtig ist: ich beschränkte mich auf ,ja“ und „nein“. Damit sind die wesentlichen Dinge gesagt. Am Telefon noch ein geknurrtes „hallo“ [sic]. In komplizierten Fällen, die aber selten waren, verwendete ich außerdem noch die Wörter ,eventuell“, ,,vielleicht“. Manchmal ließ ich mich auch zu einem „,mal abwarten“ hinreißen. Das aber schon die Ausnahme. (S. 15)

Lobeks Kommunikation mit seiner Frau beschränkt sich nur noch auf ein rudimentäres Basisvokabular, ausreichend, um alleine zu überleben, ungenügend, um sich gemeinschaftlich zu entwickeln. Die Regression wird deutlich, wenn auch das „Hallo“ nur noch animalisch geknurrt wird. Die aufs Nötigste reduzierte Kommunikation mit den Mitmenschen kann sprachlich im Roman sehr gut umgesetzt werden, denn dank des Ich-Erzählers erfährt der Leser weit mehr über Lobeks Gedanken und Gefühle als sein Umfeld, woraus sich der Ironie-Effekt ergibt, den Julia Kormann mit einfachen Worten treffend beschreibt: „Hinrich Lobek sagt häufig genau das Gegenteil vom dem, was er meint, während die Innenperspektive des Ich-Erzählers seine Worte Lügen straft" 12 . Diese Feststellung erscheint zunächst recht banal, doch das dahinterliegende Muster von geteiltem Wissen, das die Ironie erst ermöglicht, ist eine genauere Betrachtung wert. Ironie funktioniert zwischen zwei Personen nur dann, wenn die Äußerung von A von dessen Meinung abweicht und B diese Abweichung erkennt. Durch die Mitwisserschaft des Lesers an Lobeks Gedanken und Gefühlen bleibt die Ironie stets gewahrt. Dass der jeweilige Gesprächspartner im Roman sie erkennt, ist nicht notwendig.

Der Einblick in Lobeks Gedanken- und Gefühlswelt lässt jedoch nicht nur über alle Sprachkargheit hinaus die Ironie funktionieren, sondern ist auch eine wichtige Voraussetzung für den Leser, um den Protagonisten besser kennen zu lernen. Erzählperspektivisch bedingt erfährt der Leser nichts über Lobek, was nicht zuvor durch dessen Bewusstsein gefiltert und gefärbt worden ist. Ausschließlich aus Lobeks Perspektive lernt der Leser seine Geschichte, seine kleine Welt, seine großen Ängste,

12. - Julia Kormann, „Satire und Ironie in der Literatur nach 1989“, in: Volker Wehdeking (Hrsg.), Mentalitätswandel in der deutschen Literatur zur Einheit (19902000), Berlin, Erich Schmidt Verlag, 2000, S. 165-176, hier S. 176. 
sein privates Dilemma kennen. Thomas Grube beschreibt den Roman als Biografie eines Anti-Helden ${ }^{13}$, wobei dieser nie über sein Leben in Larmoyanz verfällt und selbst in depressionsähnlichen Situationen den Leser bei aller Traurigkeit zum Lächeln bringt:

Die Kreise, in denen ich mich bewegte, waren in den letzten Jahren immer kleiner, immer enger geworden. Eigentlich bewegte ich mich gar nicht mehr, sondern saß, seit meiner Abwicklung, nur noch in der Wohnung herum. Oder: ich lag einfach auf dem Sofa und starrte zum Fenster, ganze Nachmittage... Das Fenster hing schief, wahrscheinlich, weil mein Kopf schief hing. Aber den verrückten Kopf geradezurücken, dazu hatte ich nicht die Kraft. (S. 10)

Eine traurig-schöne Beschreibung, die wohl nahezu jeder Leser und das ist wichtig für die Anteilnahme - aus eigener Erfahrung so oder so ähnlich kennt. Bei aller vermittelten Traurigkeit bleibt aber die trocken humorvolle Selbstreflexion über den schiefhängenden Kopf, weswegen ihm das Fenster schräg erscheint. Dieser unerwartete Einschub des Protagonisten heitert die Situation maßgeblich auf, um sie dann zum Tiefpunkt zu bringen: die fehlende Kraft zum Geraderücken des Kopfes. Der hängende Kopf als Lebenshaltung, die sich daraus ergebende Perspektive auf die Welt ist leicht vorstellbar. Die Heiterkeit wird als Falle ausgelegt und die Melancholie tappt hinein.

Mit genau dieser Szene beginnt auch die 2001 unter der Regie von Peter Timm erschienene Verfilmung des Zimmerspringbrunnens. Die Ich-Erzählperspektive wurde vom Protagonisten gelöst und wechselt vom eher narrativen zum dramatischen Modus. Der Zuschauer beobachtet das Geschehen, ohne dabei die Perspektive einer Filmfigur einzunehmen und auch ohne Erzähler-Kommentare. So nutzt der Film die oben beschriebene ,Sofa-Szene“ als Einstieg (00:00-01:25) ${ }^{14}$. Nach dem Kameraflug über graue Plattenbausiedlungen bei trübem Wetter fährt die Kamera in Lobeks Wohnung und zum stillen Blick aus dem Fenster wird das Lied „Am Fenster“ der Ost-Berliner Gruppe „City“ gespielt. Die melancholisch-wehmütige Stimmung des Liedes, das bezeichnenderweise mit „Einmal wissen, dieses bleibt für immer“ beginnt, wird bei „Nicht die Stirne mehr am Fenster kühlen“ durch die Explosion der Kugel des Berliner Fernsehturms zerstört. Lobek reißt die Augen auf, nur ein Albtraum.

Die Kombination aus Bildern und Musik vermag Traurigkeit und Schwere besser zu vermitteln und sie ,spüren` zu lassen, als es der

13. - Grub, S. 385f.

14. - Zeitangaben zu den Filmszenen erfolgen hier und im Folgenden direkt im Text mit Angabe des Timecodes in Klammern und beziehen sich auf die Verfilmung von Peter Timm: Der Zimmerspringbrunnen, Hamburg, Senator Film, 2001. 
gedruckte Text vermag. Dies liegt vor allem an der Musik, die unmittelbar auf den Zuschauer wirkt und den Effekt der Bilder verstärkt. Verloren geht hingegen die humoristisch gestaltete Reflexionsebene und somit ein sehr wichtiges Charakteristikum Lobeks.

Während der Hund ${ }^{15}$ im Film von Frauchen Julia mit ausführlichen Streicheleinheiten begrüßt wird und Lobek nur ein leises, mitleidiges „Und ich?" entweicht (01:45), hat die Szene im Buch nicht diesen resignierenden, sondern einen frech anklagenden Charakter: ,,Ach, wenigstens mein Hassolein sagt mir guten Tag', hörte ich Julia draußen sagen. Was erwartete sie eigentlich von mir? Sollte ich etwa schwanzwedelnd zur Tür rennen? An ihr hochspringen? Ihre Hände ablecken? An ihren Taschen schnüffeln? Oder was!“ (S. 18) Der ,Buch-Lobek“ resigniert nicht, er lässt zwar lieber reden und genießt sogar sein Schweigen ${ }^{16}$, doch seine Gedanken zeigen Wachheit und bissigen Witz, der im Film abhanden kommt, was ihn wehmütig und besonders zu Beginn noch phlegmatischer erscheinen lässt. Dazu passt, dass ihn im Film kein Horoskop zur Eigeninitiative und somit zur Vertreterkarriere führt, sondern der Gang zum Arbeitsamt. Dort verschläft er im Warteflur beinahe seinen Termin und wird von seinem Sitznachbarn unsanft geweckt, was ihn letztlich zur Sachbearbeiterin mit dem Stellenangebot führt (03:30-04:15).

Der Ablauf des Einführungsseminars der Firma „PANTA RHEIn“ entspricht im Film nahezu dem der Bucherzählung, doch die stillen Eindrücke Lobeks erfährt der Zuschauer nicht. Vor allem der Auftritt und die Sprache des Firmenchefs Dr. Boldinger beeindrucken, wie der Leser in stiller Mitwisserschaft erfährt: „Ich sah gleich, daß es der Chef sein mußte! Nicht nur die äußere Erscheinung, auch sein Erscheinen selbst verriet das - schon wie er die Szene betrat, ein Auftritt! [...] eine - man kann es nicht anders sagen - künstlerische Erscheinung!“ (S. 27) Im Buch bereitet dieser Eindruck den Effekt vor, den Boldingers Sprachgebrauch auf Lobek hat:

Natürlich, nicht alles in Boldingers Rede hatte ich auf Anhieb verstanden; und die zahlreich gebrachten Details schwirrten mir noch, ohne daß ich sie recht hätte einordnen können, ziemlich zusammenhanglos im Kopf herum. Aber die Art, wie Direktor Boldinger gesprochen hatte - abgeklärt, ohne Rechthaberei, eher fragend, immer das Ganze vor Augen -, das hatte mir doch stark imponiert. (S. 30)

15. - Im Buch heißt der Hund anfangs „Hasso vom Rabenhorst“, wird später dann in „Freitag“ umbenannt. Im Film bleibt es durchgängig bei „Henry“.

16. - „Dabei, das muß ich der Vollständigkeit halber sagen, mir kam das Schweigen eigentlich entgegen. [...] Früher war mir das peinlich, wenn plötzlich das Gespräch stockte, Schweigen eintrat - damals aber begann ich, das zu genießen. Ich nickte still, schwieg." (S. 16). 
Sinnvolle Zusammenhänge kann Lobek aus den einzelnen Teilen der Rede nicht herstellen, und sein Unverständnis stellt er auch nicht in Frage, es scheint ihm ganz ,natürlich“. Geblendet und abgelenkt von der Form der Sprache, kann er sich nicht auf die Inhalte konzentrieren, gelänge es ihm, würde er sie wohl als nur scheinbare entlarven. Die ihm unverständliche Sprache ist die Sprache der Werbung und der westdeutschen Geschäftswelt. Der Schweigsame und häufig Sprachlose, der aus Überzeugung nicht redet, wenn er nichts zu sagen hat oder zweifelt, soll gerade die Sprache erlernen, die den schönen Schein zum Inhalt erhebt. Zu einer kritischen Position gegenüber dem Gesagten, besser: dem Geredeten, fehlt Lobek die notwendige Distanz. Eingelullt von schönen Worten schweigt sein sonst so bissiger Witz. Wie sehr er bei der Rede dazu neigt, Form als Inhalt zu verstehen, zeigt sich an seiner Interpretation des von Boldinger rezitierten Gedichts „Der römische Brunnen" von C. F. Meyer.

Und dann: das Gedicht!

Obwohl ich von Haus aus zu derartigen Dingen eigentlich keinen Zugang habe - das sagte mir etwas. Ich kann es nicht anders sagen! Es war, als hätte dieser Herr Meyer mir heimlich über die Schulter geschaut - wie ich vormittags zu Hause durch die Wohnung stromerte, mein Morgenlied pfeifend... wie ich die Blumen goß, das Wasser strömte... und ich - unheimlich ruhig davon wurde, steinruhig. Die Zeilen drückten aus, was ich Julia vielleicht immer hatte sagen wollen, ihr aber niemals so hatte sagen können. (ebd.)

Der Zugang zur Bedeutungsebene ist Lobek sowohl beim Gedicht als auch bei Boldingers Rede nicht möglich. Bei beiden erliegt er der Form, dem Wie der Sprache, die ihn nicht bis zum Inhalt, dem Was, vordringen lässt. Sein oberflächliches Verständnis des Gedichts führt ihn zur obigen wortwörtlichen Interpretation. Aus den Worten kann er dank ihrer Bildlichkeit noch einen Sinn konstruieren, ein Ganzes aus Boldingers Rede zu gewinnen gelingt ihm jedoch nicht und die Details schwirren zusammenhanglos in Lobeks Kopf herum. Tief gerührt vom Gedicht macht es ihn zudem auf Ungesagtes und für ihn Unsagbares aufmerksam.

Mangelnde Kommunikationsfähigkeiten Lobeks führen nicht allein zu Julias begründeter Klage über den fehlenden Austausch, worauf noch ausführlich eingegangen werden wird, sie bescheren ihm zugleich beruflich unerwartete Erfolge. Beim Rollenspiel muss sich auf Geheiß Boldingers Lobek vor den Augen der erfahrenen Kollegen in einer „kreuzgefährliche[n] Phase“ (S. 48) des Verkaufsgesprächs behaupten: „Wir haben alle, wirklich alle denkbaren Argumente gebracht, sind mithin am Ende unseres Lateins, das Pulver ist sozusagen verschossen. Was aber macht unser Kunde? - Er zögert..." (ebd.) Strüver, Leiter des Rollenspiels 
und sein späterer Kollege, mimt den Kunden und argumentiert, dass seine Frau mit dem Preis nie einverstanden wäre. Demonstrativ schüttelt er den Kopf und schiebt den Kugelschreiber weit von sich. Lobek, ihm gegenübersitzend, schaut ihn „,voller Mitgefühl“ (S. 49) an und denkt an ,,jedes Sägeblatt, jeden Sperrholzposten“ (ebd.), um die er mit Julia für seinen Hobbyraum hat ringen müssen. Sprachlos verabschiedet er sich innerlich von seiner Vertreterkarriere, zum Abschied drückt er noch mal auf die Knöpfe des kleinen Kästchens mit der Aufschrift „BUDENZAUBER“ (S. 50), das einen Zimmerspringbrunnen repräsentieren soll. Dabei kommt er zu nahe an die Fontäne und spritzt sich das Gesicht nass. , ,Jetzt müssen Sie unterschreiben, Strüver! ' hörte ich aus der Tiefe des Raums Boldingers leise Stimme. ,Wenn Sie kein Herz aus Stein haben, müssen Sie jetzt unterschreiben“" (S. 50).

Die Spielregeln der westdeutschen Geschäftswelt scheinen keine Atempausen zu erlauben, über Verlegenheit und Sprachlosigkeit wird hinweggeplappert, sophistisch wird der Kunde zur Unterschrift gelockt. Lobeks Überforderung und Hilflosigkeit werden von Boldinger als geniale Strategie gedeutet. Er habe „Tatsachen sprechen lassen“ (S. 50) und es brauche auch keine "Schnellsprechweltmeister" (S. 51), denn „der Kunde soll ja mal einen Augenblick sprachlos sein dürfen.“ (ebd.) Das Missverstehen zwischen West- und Ostdeutschen, ihren Mentalitäten und Identitäten, wird auch von Lobek aufmerksam registriert und im Stillen kommentiert: „Boldinger sah mich bewundernd an, wie man eine exotische Pflanze ansieht: ,Herrschaften, das nenne ich östliche Ruhe und meditative Kraft! Ja, Mensch, auch wir hier im Westen können von Ihnen lernen. Durchaus!“ (ebd.)

Es scheint jedoch unzulänglich, den Grund des Missverstehens allein im Aufeinandertreffen zweier grundverschiedener Sozialisierungen zu sehen. Lobek scheitert am Übergang von einer sozialistischen zu einer kapitalistischen Gesellschaft und befindet sich genau dazwischen: „Alle meine Versuche, draußen, im feindlichen Leben, wieder Fuß zu fassen, waren bis dahin ja erfolglos geblieben.“ (S. 14) In diesem Niemandsland, wo sich seine Adresse ändert, ohne dass er umzieht ${ }^{17}$, fühlt er sich allein und zurückgelassen. Es ist bezeichnend, dass er Hasso vom Rabenhorst ohne Wissen Julias heimlich umtauft: „Ich legte meine Laubsäge aus der Hand und sagte mit ruhiger Stimme: ,Du bist jetzt Freitag, Hasso! ““ (S. 18) Lobek als Robinson der Moderne ${ }^{18}$, seine Heimat- und Arbeitslosigkeit isolierten ihn, machten ihn schweigsamer. Der Schiffbrüchige hatte niemanden zum Reden, Lobek glaubt, nichts zu erzählen zu haben (vgl. S. 14).

17. - „Heimlich, über Nacht sozusagen, waren wir aus unserer Straße umgezogen worden. Sie trug jetzt einen anderen Namen.“ (S. 36f.).

18. - Vgl. auch Kormann, S. 176. 
Im Roman sind Sprache und Sprachlosigkeit, Heimat- und Heimatlosigkeit, Identität und Identitätsverlust auf das Engste miteinander verknüpft. Diese werden dem Leser vor allem durch den inneren Monolog des Ich-Erzählers, der einen ironisch-sarkastischen Schleier über ein faktisch tragisches Leben legt, immerfort ins Bewusstsein gerufen. Diese Reflexionsebene wird im Film ausgespart und durch den medienbedingten Wechsel vom Erzählen zum Darstellen, vom narrativen zum dramatischen Modus, werden ,bildschwache' Szenen wie das oben erwähnte ,stumme Verkaufsgespräch“ nicht berïcksichtigt - im Roman ist es eine der Schlüssel-Szenen.

Schweigen bringt Lobek erste ungeahnte „Erfolge“ im Beruflichen, doch im Privaten zieht ihn das Schweigen weiter in die Ehekrise. Seine Unfähigkeit, sich Julia mitzuteilen, Glück oder Angst auszudrücken, wird bereits zu Beginn des Romans deutlich. Die Freude über die Einladung zum Vertreterseminar kann Lobek ihr nicht zeigen, er bleibt isoliert in der Wohnung, legt Beethovens „Ode an die Freude“ auf den Plattenteller und sich selbst auf das Sofa (vgl. S. 20). Gefühle durch Musik auszudrücken, scheint dem Wunsch zu entsprechen, die eigene Innenwelt der Außenwelt einsehbar zu machen. Geradezu parodistisch wirkt Lobeks Verhalten, denn er produziert die Musik nicht, er reproduziert sie auf dem Plattenspieler. Er selbst liegt ruhig auf dem Sofa, die Musik, die seine Freude zum Ausdruck bringen soll, kommt also von außen und wirkt auf ihn ein. „Die Platte drehte sich. Alles drehte sich. Alles dreht sich um mich. [...] Die letzten Wochen und Monate, die ganzen Jahre (und die kaputten) zogen an mir vorüber. Sie verschwanden auf Nimmerwiedersehen [...]" (ebd.) Dennoch wirkt die Musik erleichternd, sogar befreiend, die trüben Jahre der Arbeitslosigkeit ziehen davon, und entgegen aller Gewohnheit wählte er sogar Julias Büronummer, „hatte aber, als sie sich meldete, plötzlich das Gefühl, sie sei nicht allein im Zimmer; Hugelmann ist bei ihr [...] und legte sofort wieder auf" (S. 21). Der Wunsch zu teilen ist vorhanden, die Musik gibt ihm zunächst die notwendige Kraft, doch scheitert er an den Umständen. Dank der Ich-Erzählperspektive gelingt es Sparschuh, eine ,Beziehung' zwischen Leser und Lobek zu etablieren, wohingegen die Beziehung zwischen ihm und seiner Frau solche ,Komplizenschaft ${ }^{\star}$ vermissen lässt und auch an der Eifersucht zu scheitern droht. Doch auch mit seiner Angst, sie zu verlieren, kann er sie nicht konfrontieren. Bei einem Anruf Hugelmanns geht zufällig Lobek ans Telefon:

Auf mein geknurrtes „Hallo“ hin legte die Männerstimme am anderen Ende eine verdutzte Schweigesekunde ein, um dann, ziemlich unbeeindruckt übrigens, Frau Lobek zu verlangen, Betonung auf Frau. Die Stimme hätte ohne weiteres ja auch fragen können „Darf ich bitte 
Ihre Frau sprechen?“ - aber nein, das tat sie nicht. Wahrscheinlich hatte sie Gründe dafür, in Julia etwas anderes als meine Frau zu sehen. (S. 8)

Die genau registrierte und analysierte Wortwahl des Anrufers bestätigt Lobek in seiner Eifersucht, doch zu helfen weiß er sich nicht. Anstatt das Gespräch mit Julia zu suchen, sucht er der gerade Duschenden einen Bademantel, ,damit sie sich wenigstens, wenn sie schon mit diesem Herrn spricht, etwas überhängt“ (ebd.). Um sich zu beruhigen, zieht er sich in den Hobbyraum zurück, um dort seinen Laubsägearbeiten nachzugehen. In der Verfilmung herrscht in dieser Szene (02:10-02:33) ebenfalls noch Sprachlosigkeit, doch kurz bevor Julia die Wohnung parfümiert für einen angeblichen Computerkurs verlässt, hakt Lobek nach: "So weit ich mich erinnere, war gestern auch schon Computerkurs. Ist dieser Hamann auch da?" Julias kühle Antwort: „Und wenn schon.“ (03:01-03:10). Die Eifersucht wird im Film expliziter dargestellt und die Beziehungskrise rückt mit zahlreichen Szenen, die im Buch nicht vorkommen, in den Vordergrund ${ }^{19}$.

Das Schweigen wird als Stilmittel im Roman viel stärker eingesetzt, was mit dazu führt, dass die Beziehungsprobleme des Paares zwar stets präsent sind, aber viel subtiler inszeniert werden. Im Film äußert sich Lobek in einem Streit mit Julia anklagend und mitleidsheischend zugleich: „Als Verlierer hat man's schwer, man findet kein Verständnis mehr.“ (44:22-44:25) Wenngleich auch diese Stelle durch den Reim belustigend wirkt, so ist es doch eine direkte Konfrontation. Der ,BuchLobek ' vermeidet solche Situationen, denn er erscheint sprachlich nicht mehr in der Lage, seine Frau im Gespräch zu kritisieren.

Seine Unzufriedenheit wird non-verbal kompensiert und findet Ausdruck in Protokollbuchnotizen à la „Ich kann mich mit Julias Gesamtverhalten nicht mehr einverstanden erklären“ (S. 23). Dieses Protokollbuch kommt im Roman weit häufiger zum Einsatz, ist es doch durch die Erzählerperspektive neben dem Monolog eine sehr gute Möglichkeit, dem Leser Einblicke in die Gedanken- und Gefühlswelt des Protagonisten zu geben. Grub spricht zwar von „Stasi-Manier“20

19. - Zur Veranschaulichung dieser Neugewichtung seien die neuen Szenen hier kurz aufgezählt: 1. Kollege Hamann (im Buch nur namentlich in Erscheinung tretend) lädt Julia zum Essen ein (16:05-16:51); 2. Betriebsfeier, Hamann spielt Klavier und singt, Lobek kommt dazu und betrinkt sich (30:54-35:43); 3. Julia lädt Hamann zu sich nach Hause ein, trifft dabei ungewollt auf ihren Mann (41:19-44-12); 4. Lobek sucht Julia bei ihrer Freundin (52:25-52:56); 5. Lobek und Julia treffen im Restaurant aufeinander (55:10-56:28); 6. Lobek ruft Julia bei ihrer Freundin an, geht anschließend zu ihrem Büro, verpasst sie knapp (1:03:22-1:05:18); 7. Hamann gesteht Julia seine Liebe (1:14:40-1:17:16); 8. Lobek fährt zu Julias Freundin, um sie dort zu suchen (1:17:181:18:00); 9. Julia und Lobek sehen sich am Bahnhof (1:20:50-1:23:06); 10. Julia kommt zur Versammlung von PANTHA RHEIn (1:26:00-1:28:12).

20. - Grub, S. 385. 
wenn Lobek seine Frau als „Observationsobjekt J.“ (S. 8) bezeichnet, und man kann diese und ähnliche Passagen durchaus als Parodie auf den Kontrollwahn der Staatssicherheit lesen, doch gerade als DDR-Wohnungsverwalter musste Lobek alle Probleme der Mieter und Schäden in deren Wohnungen genau dokumentieren. Und genau dies macht er: Er dokumentiert die Probleme und Schäden ihrer Beziehung. Unmittelbar helfen konnte er den Menschen schon damals nicht, die höchste Dringlichkeitsstufe für eine Reparatur lautete „nächstes Jahr“. Mit der gleichen Hilflosigkeit dokumentiert er nun routiniert und schweigend die Probleme mit Julia. Wieder im Kontrast zu diesem Verhalten steht der ,Film-Lobek“, der deutlich aktiver auftritt und Julia sogar in ihrem Büro aufsucht, denn er scheut die direkte Konfrontation keineswegs.

Eine auffällige und wichtige Gemeinsamkeit von Buch und Film ist der Morgen, an dem Lobek, neue Kräfte fühlend, erwacht. In der Hoffnung, langsam wieder Herr seines eigenen Lebens zu werden, versucht er auch Freitag mit einem Appell zu motivieren, der in Buch und Film nahezu identisch ist:

„Beweg dich doch endlich mal, du fauler Hund [...] Das Leben, Mensch, das Leben ist unendlich viel mehr als Fressen, Gassi und Glotze! Man kann nicht so wie du bloß in den Tag hineinleben. Das Leben muß doch einen Sinn haben, einen Sinn, verstehst du! ... Jeder Mensch ... überhaupt: jede Kreatur... man muß doch an etwas glauben in der Welt, verdammt noch mal!“ (S. 131 bzw. 1:09:36-1:10:03)

Durch die oben erwähnten Passagen ist deutlich die Parallele zwischen dem Hund und seinem Herrchen zu sehen: das tatenlose Hinnehmen der Ereignisse, das Hineinleben in den Tag, die sinnentleerte Existenz. In gewisser Weise führte auch Lobek lange Zeit ein sprichwörtliches ,Hundeleben“. Doch durch den beruflichen ,Erfolg' zu neuem Selbstbewusstsein gelangt (sein „Atlantis-Modell“ fand unerwartet hohen Absatz in Ost-Deutschland), ist er ein anderer geworden, womöglich ohne es zu merken (S. 130). ,[Z]u einer Hängepartie im alten Stile“ (ebd.) sollte und konnte es nicht mehr kommen.

Der neu erwachte Tatendrang richtet sich auf nur ein Ziel: „Auf der Tagesordnung stand einzig und allein: Julia!“ (S. 140) Sie ist inzwischen aus der gemeinsamen Wohnung ausgezogen, doch das Fest der Liebe steht kurz bevor und die rettende Idee scheint die Einladung zum Weihnachtsessen zu sein: „Ein großer Gedanke. Der mich ausfüllte, der keinem anderen Gedanken mehr Platz ließ.“ (S. 133) „Buch-“ und ,Film-Lobek' bereiten sich auf Julias Besuch vor: Wohnung putzen, dem Hund Kunststückchen beibringen, Essen kochen. 
Doch nur im Buch wird die Sprache und das Problem ihres Verlustes einmal mehr thematisiert. Auslöser ist Lobeks Lieblingslied „Words are very unnecessary“ (S. 140) ${ }^{21}$, in dem die vermeintliche Bedeutungslosigkeit von Worten im Vergleich zur Bedeutsamkeit von echten Gefühlen besungen wird. Inzwischen weiß er jedoch um die Relevanz der Worte und sieht wegen seines Defizits der Begegnung mit Julia sorgenvoll entgegen (S. 142). Seine Schweigsamkeit brachte beruflichen ,Erfolg', der Erfolg neues ,Selbstbewusstsein', doch die Sprache kam nicht mit zurück. Selbst die wenigen Worte, mit denen er die letzten Verkaufsgespräche bestreitet, gehen in der häuslichen Einsamkeit wieder verloren und „,bei der zu erwartenden Aussprache mit Julia würde [...] das eine oder andere sicher fehlen“ (ebd.). Der Verzweiflung nahe, sagt er, zur Selbstvergewisserung, „Tisch zum Tisch, Glas zum Glas, Zigarette zur Zigarette, Asche zur Asche, Staub zum Staub“ (ebd.) und um ein „menschliches Elementargeräusch zu hören" (ebd.), lacht er die Küchenwand an. Es ist einerseits die soziale Isolation eines Robinson Crusoe, aufgrund derer er seine Sprache durch kontinuierlichen Nichtgebrauch zu verlieren droht, andrerseits droht sein Leben ohne Julia wieder zu einer sinnentleerten Existenz zu werden.

Zum geplanten Weihnachtsessen kommt es nicht, knapp verpassen sie sich und in der Wohnung findet Lobek ihre Abschiedskarte: „,Ich hab Dich sehr lieb!' stand da; ich setzte mich hin. Und weiter unten, unter dem goldigen Tannenzweig, las ich die Worte: ,Aber ich kann nicht mir Dir leben! - Julia.“" (S. 144 bzw. 1:13:45-1:13:55). Nach anfänglicher Niedergeschlagenheit raffen sich ,Buch-" und ,Film-Lobek' wieder auf, beide mit der Absicht, das sich abzeichnende Ende der Ehe nicht mehr als unabwendbar hinzunehmen.

Im Film wird Julia gekündigt, Lobek beendet seine vielversprechende Vertreterkarriere und beide widmen sich ihrer Beziehung. Dieses unerwartete Ende ist wenig plausibel und kaum aus der Handlung erklärbar zumal auch noch Julia aktiv wird und zu ihrem Mann zurückkehrt. Im Roman wird ein nahezu gegenteiliges Ende erzählt: Tatkräftig springt Lobek vom Sofa auf und appelliert entschlossen an Freitag: „Los, auf! Es wird Zeit, mein Lieber, daß wir unseren hübschen kleinen Robinson-Club hier auflösen. Marsch!“ (S. 145) Durch das Schnüffeln an Julias Nachthemd soll Freitag ihre Spur aufnehmen, woraufhin er winselt und dann bellt. „Er hatte, im Unterschied zu mir, seine Sprache wiedergefunden.“ (S. 146) Als beide durch das Treppenhaus eilen, überkommt es Lobek, auch vermeintlich andere Robinsons zum Aufgeben ihres Insulaner-Daseins anzuspornen. „Kommt doch endlich

21. - Offiziell lautet der Titel „Enjoy the silence“ und gilt als erfolgreichstes Lied der Gruppe „Depeche Mode“. 
raus aus euren Höhlen.“ [...] „Bitte! Ihr Idioten!“ (ebd.) Im Rausch des eigenen Aufbruchs möchte er ,verhauste" Leidensgenossen mitreißen, sie ebenfalls retten. Diese neue Aufgewühltheit in Lobek weiß der Text sofort ironisch zu unterwandern: „Ich wollte die Haustür krachend ins Schloß werfen, aber die Pneumatik dämpfte schnaufend den wütenden Schwung.“ (ebd.) Die Ironie erlaubt Distanz zum Geschehen, Witz und Reiz liegen im Schwebezustand der Erzählung, im Halten der Balance zwischen Tragik und Komik.

Es ist naheliegend, dass ein solcher Romanstoff in diesem tragischironischen Stil kein ,Happy End“ verträgt, es würde den Roman selbst karikieren. Lobek und Freitag harren über eine Woche am Bahnhof aus, doch das ersehnte Wiedersehen mit Julia bleibt aus. In dieser Zeit bleiben sie jedoch nicht allein, denn ,[a]n der Wand gegenüber lauerte eine graue laute Männerschar. Sie sahen aus wie Ali Babas vierzig Räuber; aber es waren weniger. Stadtstreicher, Penner, Obdachlose, wie man wohl annehmen mußte." (S. 150) Es hat Symbolkraft, wenn die am Rande der Gesellschaft Lebenden am Ende des Jahres zu Lobeks Notgemeinschaft werden, doch der aufgesetzte biblische Sprachgestus und sein Brechen ironisieren auch diese Situation: „Ich öffnete meinen Rucksack und sprach: ,Hört! Ich will mein Brot mit euch teilen.' Sie aber sprachen zu mir: ,Mann, warum sagste'n det nicht gleich!' Und so geschah es. [...] In ihren Gesichtern stand Zufriedenheit ob der Gaben." (S. 152) Hier gelingt Lobek, was ihm mit Julia unmöglich war: Das sprachliche Mitteilen wird zum Teilen des Brotes, zum Aufbau einer wenn auch nur für wenige Tage bestehenden Gemeinschaft. Am Neujahrstag verlässt er sie bei Sonnenaufgang mit Freitag, der Aufbruch ist nicht enthusiastisch, doch dafür entschieden. „Ich zog Freitag an der Leine. ,Na, los, komm schon!' Komm.“ (S. 159) Das letzte Wort, „Komm“, durch eine Leerzeile im Roman abgesetzt, nur noch gedacht, nur hörbar für den Leser, eine Ermahnung und Aufforderung an sich selbst. Doch bei aller Traurigkeit der Situation, Lobeks Humor geht nicht verloren, und so kommentiert er den Sonnenaufgang trocken: „Daß die sich das überhaupt noch traute... Immerhin, sie war schamrot!“ (ebd.)

\section{Fazit}

Von der Stärke des Romans, den ,subtilen und komplexen Ironien der Sprache" 22 , konnte nur eine kleine Auswahl berücksichtigt werden, die Der Zimmerspringbrunnen dem Leser großzügig anbietet. Durch die Perspektive des Ich-Erzählers und den burlesken Sprachgebrauch wird ein ironisch-sarkastischer Schleier über den traurigen Alltag geworfen. Bedeutungszusammenhänge werden gelöst, neu geordnet bzw. bleiben

22. - Monaco, S. 47. 
in der Schwebe, und der Text balanciert zwischen Tragik und Komik. Die selbstdiagnostizierte und häufig thematisierte Sprachkrise Lobeks steigert das ironische Spiel mit Bedeutungen, das der Autor treibt. Dabei ermöglicht der Roman das Lachen über die Oberfläche, aber auch das Nachdenken über die Tiefe des Witzes. Wenn beim Aufräumen des Bücherregals festgestellt wird, dass ,durch die Aussortierung der Heimwerkerliteratur [...] die Bereiche SF [Science-Fiction] und ML [Marxismus/Leninismus] vollkommen ineinandergerutscht" (S. 139) sind, deutet dies auf eine weit tiefere Bedeutungsebene.

Peter Timms Verfilmung ist sehr frei nach dem Romanvorbild gestaltet. Weder bedient er sich des Ich-Erzählers noch versucht er die Ironie der Vorlage in den Film zu übertragen. Eine Sprach- und Identitätskrise durchleidet Lobek nicht, innere unausgesprochene Konflikte hat er nicht. Es wäre gewiss möglich gewesen, die Stärken der Vorlage auch für den Film zu nutzen, doch Timm entwickelt neue Szenen und setzt neue Akzente. Die Sprachverliebtheit des Romans geht verloren, der Protagonist verliert an Tiefe und erscheint als sympathischer Verlierer, der auch mal Glück hat. Die filmisch leicht zu inszenierende Beziehungsproblematik wird ausgewalzt und dominiert die Handlung. Als selbstständige Arbeit gesehen ist der Film unterhaltsam und kurzweilig, jedoch weniger tiefgründig als der Roman. Der humorvoll reflektierende Erzähler des Romans lässt den Leser eine weit komplexere Welt sehen, als sie der Film dem Zuschauer zeigt. Die Fähigkeit zum Spiel mit Worten ist die größte Stärke des gedruckten Textes. Diese Stärke ausspielend, zeigt Sparschuhs Roman Merkmale von Poesie, indem er, um mit Monacos Worten zu schließen, ,seine Aufmerksamkeit verstärkt auf sich selbst richtet und sein eigenes Material zelebriert: die Sprache“23. Und dies von einem Filmwissenschaftler. 


\section{Literatur}

Grub, Frank Thomas, ,, Wende “ und „Einheit“ im Spiegel der deutschsprachigen Literatur, Bd. 1 Untersuchungen, Berlin, de Gruyter, 2003.

Kormann, Julia, „Satire und Ironie in der Literatur nach 1989“, in: Volker Wehdeking (Hrsg.): Mentalitätswandel in der deutschen Literatur zur Einheit (1990-2000), Berlin, Erich Schmidt Verlag, 2000, S. 165-176.

Monaco, James, Film verstehen. Kunst, Technik, Sprache, Geschichte und Theorie des Films und der neuen Medien, Hamburg, Rowohlt, 5. Auflage, 2004.

Sparschuh, Jens, Der Zimmerspringbrunnen, Berlin, Goldmann Verlag, 1997.

Twark, Jill E., Humor, Satire and Identity: Eastern German Literature in the 1990s, Berlin, de Gruyter, 2007.

\section{Film}

Timm, Peter, Der Zimmerspringbrunnen, Senator Film, Hamburg, 2001. 
